\title{
Apego parental y dependencia emocional en mujeres del distrito de Túcume - Lambayeque, Perú
}

\author{
Parental attachment and emotional dependence on women in the Túcume \\ district - Lambayeque, Peru
}

\author{
Dajhana G. Gómez Guevara \\ psicologadajhanagomez@yahoo.com \\ Universidad Ricardo Palma, Perú
}

Gustavo X. Sánchez Manayay

Dina M. Calonge De la Piedra

tavinho_21@hotmail.com

marisolcalonge@hotmail.com

Universidad Señor de Sipán, Perú

\section{RESUMEN}

El propósito del presente estudio fue determinar la relación entre el apego parental y la dependencia emocional. La muestra estuvo formada por 110 mujeres con edades comprendidas entre 18 y 36 años del distrito de Túcume- Lambayeque en el centro poblado la Raya en Perú. Para establecer la relación se aplicó un diseño transversal correlacional en el cual se midió el apego emocional y la dependencia emocional, utilizando el Cuestionario de Lazos Parentales - Parental Bonding Instrument (PBI Parker, Tuplin y Brown, 1979) y el Inventario de Dependencia Emocional (IDE Aiquipa Tello, 2012). Se encontró una relación significativa entre el apego parental y la dependencia emocional. En cuanto al análisis correlacional de las variables de estudio se encontró correlación altamente significativa entre la dimensión cuidado paterno y la dimensión prioridad de la pareja; sobreprotección materna con miedo a la soledad; sobreprotección paterna con las dimensiones prioridad a la pareja; deseos de control y dominio; subordinación y sumisión a un nivel de significancia, las mujeres de la muestra manifestaron comportamientos $y$ conductas orientadas a mantenerse en relaciones inapropiadas perjudicando su autonomía emocional.

Palabras clave: apego parental; dependencia emocional; Parental Bonding Instrument; Inventario de dependencia emocional

\begin{abstract}
The purpose of the present study was to determine the relationship between parental attachment and emotional dependence. The sample consisted of 110 women between the ages of 18 and 36 from the TucumeLambayeque district in the La Raya populated center in Peru. To establish the relationship, a correlational cross-sectional design was applied in which emotional attachment and emotional dependence were measured, using the Parental Relationships - Parental Bonding Instrument Questionnaire (PBI Parker, Tuplin and Brown, 1979) and the Emotional Dependence Inventory (IDE Aiquipa Tello, 2012). A significant relationship was found between parental attachment and emotional dependence. Regarding the correlational analysis of the study variables, a highly significant correlation was found between the parental care dimension and the couple's priority dimension; maternal overprotection with fear of loneliness; parental overprotection with the priority dimensions to the couple; desires for control and mastery; subordination and submission to a level of significance, the women in the sample showed behaviors and behaviors aimed at maintaining inappropriate relationships damaging their emotional autonomy.
\end{abstract}

Key words: parental attachment; emotional dependence; Parental Bonding Instrument; Inventory of emotional dependence 


\section{INTRODUCCIÓN}

En el transcurso de la vida de un ser humano las relaciones con las otras personas del entorno van experimentando cambios. El apego es la vinculación afectiva más temprana, que se establece entre el niño y el cuidador más cercano, y es la que permite aprender a regular el sistema emocional. (Fonagy, 2004). Al respecto Bowlby (1969) enfatiza que el apego es el lazo afectivo que se establece con otra persona considerada importante para el bienestar físico y psicológico; determinado por la necesidad de proximidad física y emocional. Estos primeros vínculos afectivos de la infancia pueden ser positivos generando sentimientos de seguridad, afecto y confianza o negativos caracterizados por la inseguridad, abandono y miedo.

En consecuencia la relación afectiva Paterno- filial es la base de los sentimientos de seguridad o inseguridad que presentan los individuos en su vida adulta. (Lafuente, 2003). La función principal del apego adulto sigue siendo la misma que la del apego infantil proporcionar protección y seguridad. Tanto Bowlby (1989) como Ainsworth (1989) apuntaron la importancia que tienen los vínculos de apego establecidos con los padres durante la infancia para el establecimiento de posteriores relaciones afectivas. Es así como el apego infantil dimensiona las relaciones interpersonales durante toda la vida. Unas relaciones de apego seguro durante la infancia le otorgan al adulto mayores capacidades para establecer relaciones afectivas positivas con sus iguales. Por el contrario unas relaciones de apego inseguro durante la infancia podrían originar, en la adultez, relaciones afectivas negativas caracterizadas por la dependencia emocional.

Castelló (2000) define la dependencia emocional como "un patrón persistente de necesidades emocionales insatisfechas que se intentan cubrir desadaptativamente con otras personas". La esencia de este problema es que la persona dependiente presenta una frustración o una insatisfacción en su área afectiva que pretende compensar centrándose en sus relaciones de pareja. Cuando esto ocurre la relación que se genera se caracteriza por la ansiedad, sensación de vacío y miedo al abandono. Esta conducta no solo aplica a las parejas sino que se experimenta de similar manera en relaciones amistosas y familiares.

Las características de la dependencia emocional según Castelló (2012) son:

1. Prioridad de la pareja sobre cualquier otra cosa: El dependiente emocional pone a su relación por encima de todo, incluso de él mismo, de su trabajo, de sus hijos, de sus gustos y aficiones. Termina desdibujando su vida hasta que prácticamente se convierte en su sombra.

2. Voracidad afectiva o deseo de acceso constante: El dependiente emocional puede decidir por sí mismo cómo, cuándo y de qué forma contacta con su pareja. El contacto puede ser muy frecuente y excesivo, hasta el punto que pueda originar problemas en su entorno laboral, familiar o social.

3. Tendencia a la exclusividad en las relaciones: Esto ocurre tanto en las relaciones de pareja como con las amistades de estas personas. El dependiente emocional se siente más cómodo hablando con un único amigo que en un grupo numeroso, implica una cierta falta de construcción personal, se aísla de su entorno, por dedicarse por entero a su pareja y espera que el otro actúe de la misma forma.

4. Prioridad de la pareja: Esta característica ilustra a la perfección la similitud con otras adicciones, ya que en ambos fenómenos es el objeto de la adicción lo 
que se convierte en el centro de la existencia del individuo, y todo lo demás queda al margen. La pareja del dependiente emocional ocupa continuamente su pensamiento, sus sentimientos y su comportamiento, descuidándose así aspectos como trabajo, hijos, familia, amigos, aficiones personales.

5. Idealización excesiva: El otro se convierte con el tiempo en alguien sobrevalorado pudiendo distorsionar sus méritos y capacidades. La idealización es el fundamento de su necesidad patológica por el compañero de exclusividad, deseo de acceso constante y prioridad.

6. Subordinación en las relaciones de pareja: Es un medio para preservar la relación a toda costa, hay que señalar que la mencionada subordinación y el desequilibrio existente en la pareja es en ocasiones motivo de consulta, sobre todo en casos de dependencia emocional, el dependiente entra en pánico ante la ruptura y esto puede ocasionarle el padecimiento de una psicopatología.

7. Sucesión ininterrumpida de parejas: Del rasgo anterior se desprende que en muchas ocasiones los dependientes encadenan una relación tras otra, aunque no todas se ajustan necesariamente al modelo desequilibrado de pareja que es el que ellos desean, de hecho, pueden tener "relaciones de transición" con personas hacia las que no tengan sentimientos fuertes, para así paliar su sufrimiento por la soledad y estar en mejor disposición de encontrar a otro individuo verdaderamente "interesante".

Una de las consecuencias de la dependencia emocional en las mujeres es que éstas se convierten en víctimas de sus parejas, al respecto en una entrevista con el Sub Oficial Superior Zapata(2015) quien se desempeña como Jefe Sectorial en la Defensoría de la Mujer en la Comisaria Sectorial de Lambayeque, este funcionario refiere que durante el año 2015 se incrementó el abuso en mujeres víctimas de maltrato con agresión física, psicológica y sexual por parte de sus parejas, en un $30 \%$, desde enero del 2014, hasta la fecha. Asimismo declaró que solo un $2 \%$ le dan continuidad al proceso judicial y por ende el 98\% de estas mujeres abandona el proceso iniciado, incluso refiere que al momento de dar información se niegan a dar los datos generales de sus agresores evidenciando protección hacia ellos.

Este comportamiento de las mujeres, descrito por Zapata, lo explica Castelló (2010), quien afirma que los dependientes afectivos después de una ruptura, vivida como un acontecimiento catastrófico, intentan reanudar la relación por nefasta que haya sido o bien buscan a otra persona que cubra su necesidad extrema de estar acompañados de alguien. Esta preocupación excesiva en mantener la relación interpersonal produce una ausencia de felicidad y confianza proclive de una ruptura inmediata. Por otro lado, el rechazo produce dependencia interpersonal.

Al revisar las estadísticas de los problemas de las mujeres atendidas, en el Servicio Psicológico del Centro de Prácticas Pre-profesionales de Lambayeque, se observa un incremento en los casos de maltrato psicológico, físico y sexual, por parte de sus parejas y de forma reiterada. Estas mujeres demuestran una necesidad afectiva $y$ subordinada, ante el ser que las lastima y las humilla. Se mantienen siempre a la expectativa de un cambio favorable de la conducta del agresor, hecho que aumenta el riesgo de su situación de vulnerabilidad. Estas mujeres en algunas etapas de su infancia 
podrían haber experimentado carencias afectivas, posteriormente, en la adolescencia y adultez conllevaría a manifestarse en sentimientos de inferioridad, inseguridad, pérdida de autonomía lo cual contribuye a que permanezcan en relaciones insanas.

Con base en lo anterior se fundamenta el interés en esta temática, ya que la comprensión de la relación entre el apego parental y la dependencia emocional, permitirán disponer de conocimientos apropiados que sean fundamento teórico para el desarrollo de propuestas de prevención, promoción e intervención en esta población. Asimismo puede originarse un equipo interdisciplinario para poner en práctica un plan de trabajo con los profesionales de las diferentes áreas de la Psicología interesados en esta situación.

Con base en los planteamientos anteriores esta investigación enuncia el siguiente problema ¿Cuál es la relación entre el apego parental y la dependencia emocional en mujeres del centro poblado "La Raya" del distrito de Túcume - Lambayeque, Perú? Como objetivo busca determina si existe relación significativa entre apego parental y dependencia emocional en mujeres del centro poblado "La Raya" del distrito de Túcume Lambayeque, Perú.

\section{MÉTODO}

La investigación se clasifica como correlacional, tiene como finalidad conocer la relación o grado de asociación que exista entre dos o más conceptos, categorías o variables en un contexto en particular. En este trabajo se estableció la relación entre el apego parental y la dependencia emocional. El diseño es de campo, transversal ya que se toman los datos directamente de los sujetos investigados en su ambiente natural donde ocurren los hechos, sin manipulación de variables, la medición se realiza en un solo momento

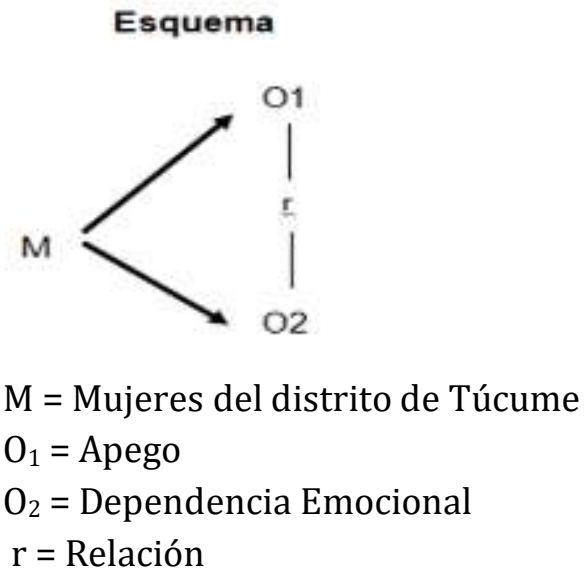

Gráfico 1. Esquema del diseño de investigación 
La investigación se realizó con una muestra no probabilística o denominada como muestra dirigida, que viene a ser un subgrupo de la población en la que la relección de los elementos no depende de la probabilidad, sino de las características de la investigación. La muestra quedó conformada con un total de 110 mujeres, entre 18 y 36 años, con una relación de pareja actual y por lo menos una relación de pareja en el pasado y residentes del centro poblado "La Raya" del distrito Túcume - Lambayeque.

Las variables en esta investigación son el apego parental y la dependencia emocional, quedan definidas:

Apego parental: Es el vínculo afectivo que se establece con otra persona considerada importante para el bienestar físico y psicológico. Esta caracterizado por una necesidad de proximidad física y emocional (Bowlby, 1969). Cuando se hace referencia en el término al apego parental se circunscribe al vínculo con los padres.

Dependencia Emocional: Es el patrón persistente de necesidades emocionales insatisfechas que se intentan cubrir desadaptativamente con otras personas, el dependiente presenta una frustración o una insatisfacción en su área afectiva que pretende compensar centrándose preferentemente en sus relaciones de pareja Castelló (2012).

\section{Técnicas e Instrumentos}

Se utilizan técnicas psicométricas para medir elementos de la personalidad de los individuos de la población en estudio. La evaluación psicológica: es una medida objetiva y estandarizada de una muestra de conducta.

El instrumento para medir el apego parental es el desarrollado por Parker, Tupling y Brown (1979) el cual permite medir el vínculo entre padres e hijos, su denominación en inglés Parental Bonding Instrument (PBI). El test original consta de 25 afirmaciones, las cuales constituyen dos escalas, que en el presente estudio se denominan, afecto (12 ítems) y control (13 ítems).

El factor de afecto, que establece la escala del mismo nombre, está definido como un continuo que va desde el polo de cuidado, contención emocional, empatía y cercanía, hasta el polo de frialdad emotiva, indiferencia $\mathrm{y}$ negligencia.

El factor control, equivale a la segunda escala se define con la percepción de estrategias de sobreprotección, intrusión, constricción, contacto excesivo, infantilización y prevención de la conducta autónoma. Como característica de una dimensión cada una puede ser medida de manera independiente, obteniendo así un puntaje para afecto y otro para control. Para el análisis de los resultados se pueden determinar cuatro tipos de vínculos parentales de acuerdo a los puntajes en Afecto y Control.

Vínculo Óptimo: son aquellos padres que obtienen puntajes altos en la escala de afecto y bajos en la de control. Se caracterizan por ser afectuosos, empáticos y contenedores emocionales, estos padres favorecen la independencia y la autonomía.

Vínculo Ausente o Débil: son aquellos padres que obtienen puntajes bajos en afecto y bajos en control. Se caracterizan por presentar frialdad emotiva, indiferencia y poca respuesta empática a las necesidades del niño.

Constricción Cariñosa: son aquellos padres que puntúan con alto puntaje en afecto y alto en control. Se caracterizan por presentar afectuosidad, contención emocional, empatía y cercanía, por un lado, y al mismo tiempo son controladores, intrusivos, tienen un contacto excesivo, 
infantilizan y previenen la conducta autónoma de sus hijos.

Control Sin Afecto: son aquellos padres que puntúan afecto bajo y alto control. Se caracterizan por presentar frialdad emotiva, indiferencia y negligencia, al mismo tiempo que son controladores, intrusivos, tienen un contacto excesivo, infantilizan y previenen la conducta autónoma.

La dependencia emocional se mide con el Inventario de Dependencia Emocional (IDE) de Aiquipa Tello (2012) con las siguientes dimensiones:

1. Miedo a la ruptura (MR): Es el temor que se experimenta ante la sola idea de disolución de la relación de pareja. Por ello, se adoptan conductas y comportamientos orientados a mantener la relación, sin importar lo perjudicial que pueda ser para la persona afecta de dependencia emocional. También hace referencia a la negación por parte del sujeto cuando se hace realidad una ruptura, ejerciendo continuos intentos $\mathrm{y}$ exhortaciones para reanudar la relación.

2. Miedo e intolerancia a la soledad (MIS): consiste en la tendencia a adoptar cualquier tipo de conductas para mantenerse ocupados todo el tiempo posible para no encontrarse con ellos mismos o realizar intentos desesperados por retomar la relación o buscar otra relación lo más pronto posible si ha terminado una.

3. Prioridad de la pareja (PP): es la tendencia a mantener en primero lugar de importancia a la pareja sobre cualquier otro aspecto o personas. Hay una consideración excesiva de la pareja, identificada como el centro de existencia, el sentido de vida, el objeto predilecto de atención.

4. Necesidad de acceso a la pareja NAP): Deseos de tener presente a la pareja en todo momento, ya sea físicamente o a través del teléfono o las redes sociales.

5. Deseos de exclusividad (DEX): Tendencia a enfocarse en la pareja y aislarse paulatinamente del entorno, acompañada deseos de reciprocidad de esta conducta por la pareja.

6. Subordinación y sumisión (SS): Sobreestimación de las conductas, pensamientos, sentimientos e intereses de la pareja, acompañada sentimientos de inferioridad y desprecio en el dependiente emocional.

7. Deseo de control y dominio (DCD): Es la Búsqueda activa de atención y afecto para captar el control de la relación de pareja, a fin de asegurar su permanencia.

\section{Hipótesis}

Las hipótesis de investigación $\left(\mathrm{H}_{\mathrm{i}}\right)$ relacionan las dos variables en estudio y las Hipótesis nulas la $\left(\mathrm{H}_{0}\right)$ la rechazan.

\section{Hipótesis General}

$\mathbf{H}_{\mathbf{i}}$. Existe relación entre el apego parental y la dependencia emocional en mujeres del Centro Poblado "La Raya" del distrito de Túcume - Lambayeque en el 2016.

Ho. No existe relación entre apego parental y la dependencia emocional en mujeres del centro poblado "La Raya" distrito de Túcume - Lambayeque en el 2016.

\section{Hipótesis Específicas}

Estas hipótesis establecen la relación entre las dimensiones de apego parental y dependencia emocional. Por lo cual se escriben en forma abreviada mencionando cada una de las dimensiones. Así mismo se obvia escribir las hipótesis nulas $\left(\mathrm{H}_{0}\right)$ que son la contraparte o negación de las hipótesis de investigación, que no existe relación. 
$\mathbf{H}_{\mathbf{i 1}}$. Existe relación entre la dimensión cuidado materno de apego parental y cada una de las dimensiones de dependencia emocional: miedo a la ruptura, miedo e intolerancia a la soledad, prioridad de la pareja, necesidad de acceso a la pareja, deseos de exclusividad, subordinación y sumisión, deseo de control y dominio en mujeres del centro poblado "La Raya" del distrito de Túcume - Lambayeque.

$\mathbf{H}_{\mathbf{i} 2 \text {. Existe la relación entre la dimensión }}$ cuidado paterno de apego parental y cada una de las dimensiones de dependencia emocional: miedo a la ruptura, miedo e intolerancia a la soledad, prioridad de la pareja, necesidad de acceso a la pareja, deseos de exclusividad, subordinación y sumisión, deseo de control y dominio en mujeres del centro poblado "La Raya" del distrito de Túcume - Lambayeque.

$\mathbf{H}_{\mathbf{i} 3 .}$ Existe relación entre la dimensión sobreprotección materna de apego parental y cada una de las dimensiones de dependencia emocional: miedo a la ruptura, miedo e intolerancia a la soledad, prioridad de la pareja, necesidad de acceso a la pareja, deseos de exclusividad, subordinación y sumisión, deseo de control y dominio en mujeres del centro poblado "La Raya" del distrito de Túcume - Lambayeque.

$\mathbf{H}_{\mathbf{i} 4}$. Existe relación entre la dimensión sobreprotección paterna de apego parental y cada una de las dimensiones de dependencia emocional: miedo a la ruptura, miedo e intolerancia a la soledad, prioridad de la pareja, necesidad de acceso a la pareja, deseos de exclusividad, subordinación y sumisión, deseo de control y dominio en mujeres del centro poblado "La Raya" del distrito de Túcume - Lambayeque.

\section{RESULTADOS}

A continuación se presentan los resultados de esta investigación. Se reportan en primer lugar los dos análisis descriptivos el apego parental y la dependencia emocional. Los resultados de la correlación de Pearson de cada dimensión de apego parental con las dimensiones de dependencia emocional. La correlación de Pearson general para determinar si existe relación entre las variables del estudio. 


\section{Descriptivo Apego parental}

\section{Cuadro 1. Análisis descriptivo de la variable apego parental}

\begin{tabular}{cccc}
\hline \multicolumn{2}{c}{ Apego parental } & F & \% \\
\hline \multirow{4}{*}{ Cuidado materno } & Alto & 31 & 28.2 \\
& Medio & 47 & 42.7 \\
& Bajo & 32 & 29.1 \\
& Total & 110 & 100 \\
& Alto & 31 & 28.2 \\
Cuidado paterno & Medio & 40 & 36.4 \\
& Bajo & 39 & 35.5 \\
Sobreprotección & Total & 110 & 110 \\
materna & Alto & 33 & 30 \\
& Medio & 42 & 38.2 \\
Sobreprotección & Bajo & 35 & 31.8 \\
paterna & Total & 110 & 100 \\
& Alto & 31 & 28.2 \\
& Medio & 44 & 40 \\
\cline { 2 - 4 } & Bajo & 35 & 31.8 \\
\hline Apego Parental & Total & 110 & 100 \\
\hline Total & Alto & 33 & 30 \\
& Medio & 42 & 38.2 \\
\hline & Bajo & 35 & 31.8 \\
\hline & Total & 110 & 100 \\
\hline
\end{tabular}

Nota: Población de 110 mujeres. 


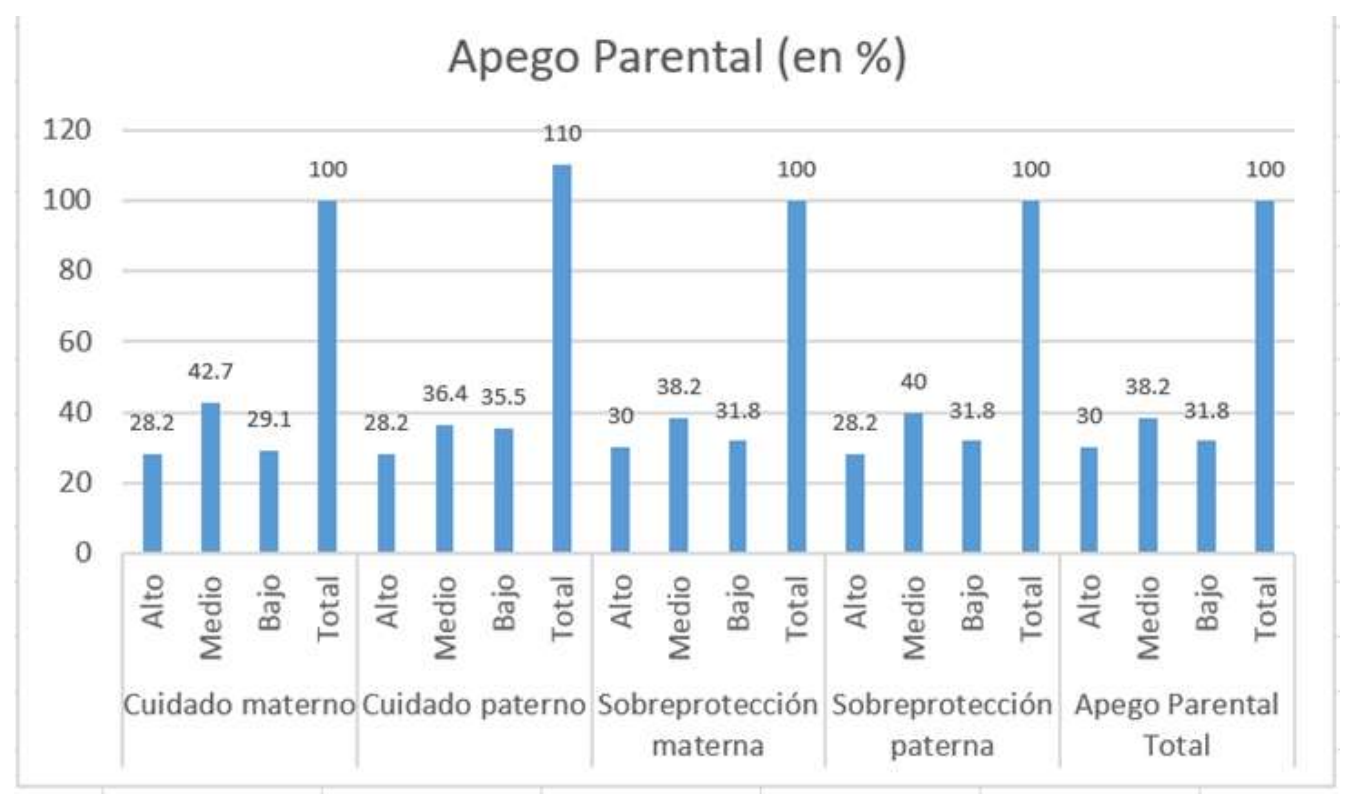

Gráfico 2. Descriptivo de apego parental

El nivel preponderante en la población, respecto a los resultados de apego parental es Medio, 38.2\%, seguido del nivel Bajo, 31.8\% y por último el nivel Alto 30\%. La población de mujeres en un $68,2 \%$ se encuentra entre los valores de apego parental medio y alto. La función del apego en los adultos es la misma que en los niños conseguir la seguridad. (Hazan y Shaver, 1994). Las mujeres de la muestra tienen un apego parental medio. 


\section{Descriptivo dependencia emocional}

Cuadro 2. Análisis descriptivo de la variable dependencia emocional

\begin{tabular}{|c|c|c|c|}
\hline Dependencia e & cional & $\mathbf{F}$ & $\%$ \\
\hline \multirow{4}{*}{$\begin{array}{l}\text { Miedo a la } \\
\text { ruptura }\end{array}$} & Alto & 31 & 28.2 \\
\hline & Medio & 45 & 40.9 \\
\hline & Bajo & 34 & 30.9 \\
\hline & Total & 110 & 100 \\
\hline \multirow{4}{*}{$\begin{array}{l}\text { Miedo a la } \\
\text { soledad }\end{array}$} & Alto & 29 & 26.4 \\
\hline & Medio & 53 & 48.2 \\
\hline & Bajo & 28 & 25.5 \\
\hline & Total & 110 & 100 \\
\hline \multirow{4}{*}{$\begin{array}{c}\text { Prioridad de la } \\
\text { pareja }\end{array}$} & Alto & 29 & 26.4 \\
\hline & Medio & 55 & 50 \\
\hline & Bajo & 26 & 23.6 \\
\hline & Total & 110 & 100 \\
\hline \multirow{4}{*}{$\begin{array}{c}\text { Necesidad de } \\
\text { acceso a la pareja }\end{array}$} & Alto & 30 & 27.3 \\
\hline & Medio & 49 & 44.5 \\
\hline & Bajo & 31 & 28.2 \\
\hline & Total & 110 & 100 \\
\hline \multirow{4}{*}{$\begin{array}{c}\text { Deseos de } \\
\text { exclusividad }\end{array}$} & Alto & 32 & 29.1 \\
\hline & Medio & 28 & 25.5 \\
\hline & Bajo & 50 & 45.5 \\
\hline & Total & 110 & 100 \\
\hline \multirow{4}{*}{$\begin{array}{l}\text { Subordinación y } \\
\text { Sumisión }\end{array}$} & Alto & 34 & 30.9 \\
\hline & Medio & 35 & 31.8 \\
\hline & Bajo & 41 & 37.3 \\
\hline & Total & 110 & 100 \\
\hline \multirow{4}{*}{$\begin{array}{l}\text { Deseo de control } \\
\text { y dominio }\end{array}$} & Alto & 33 & 30 \\
\hline & Medio & 33 & 30 \\
\hline & Bajo & 44 & 40 \\
\hline & Total & 110 & 100 \\
\hline \multirow{4}{*}{$\begin{array}{c}\text { Dependencia } \\
\text { emocional Total }\end{array}$} & Alto & 32 & 29.1 \\
\hline & Medio & 41 & 37.3 \\
\hline & Bajo & 37 & 33.6 \\
\hline & Total & 110 & 100 \\
\hline
\end{tabular}

El nivel dominante en la población, respecto a los resultados de dependencia emocional es medio, 37.3\%, seguido del nivel Bajo, 33.6\% y por último el nivel Alto, 29.1\%. La dependencia emocional también se ubica la mayor frecuencia de los datos en el nivel medio. 


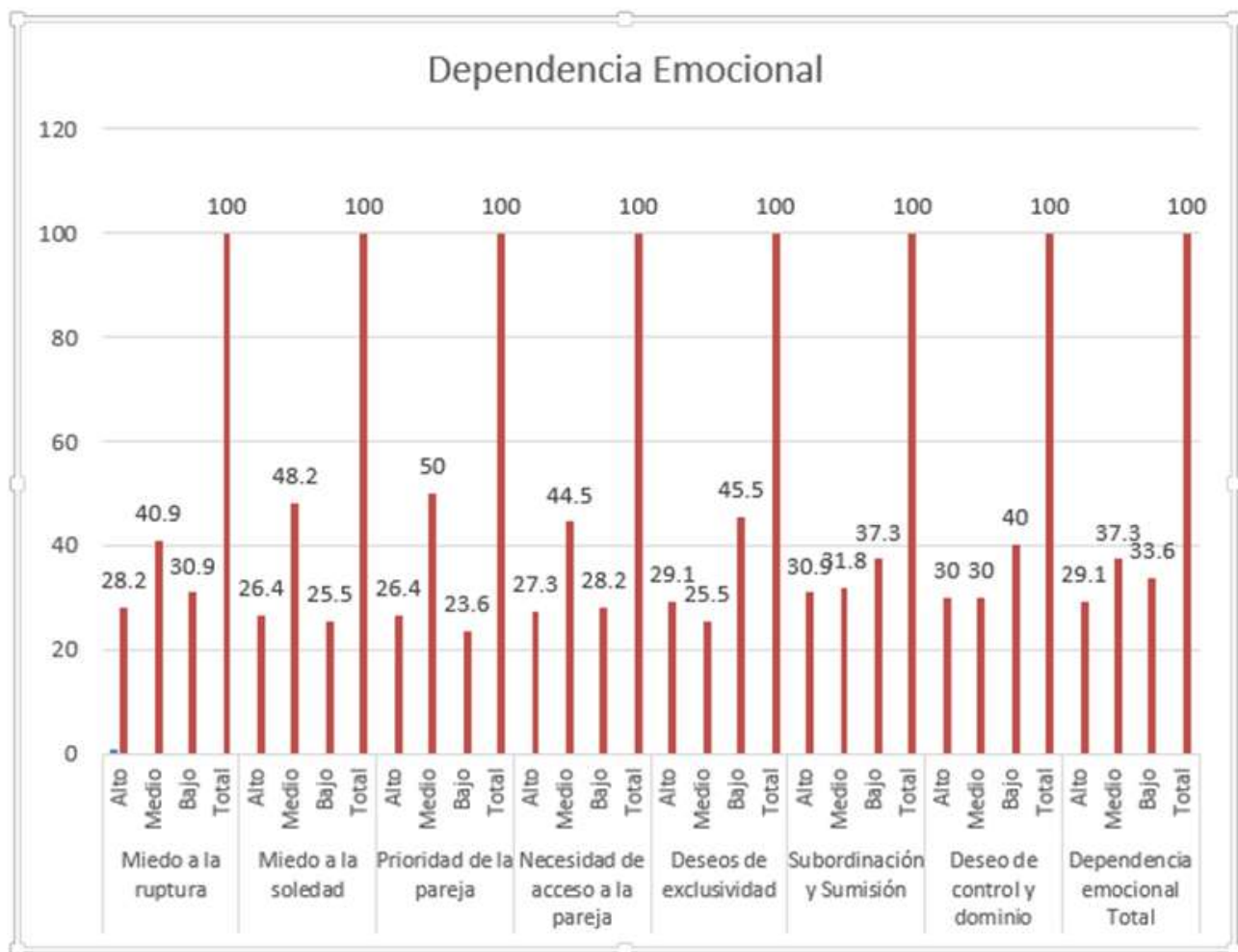

Gráfico 3. Descriptivo dependencia emocional

\section{Relación apego parental y dependencia emocional}

Cuadro 3. Análisis de relación entre apego parental y dependencia emocional

\begin{tabular}{cccccccccc}
\hline \multicolumn{10}{c}{ Apego parental } \\
\hline $\begin{array}{c}\text { Dependencia } \\
\text { Emocional }\end{array}$ & \multicolumn{2}{c}{ Bajo } & \multicolumn{2}{c}{ Medio } & \multicolumn{2}{c}{ Alto } & \multicolumn{2}{c}{ Total } \\
\hline Bajo & F & \% & F & \% & F & \% & F & \% \\
Medio & 10 & 9.1 & 13 & 11.8 & 9 & 8.2 & 32 & 29.1 \\
Alto & 11 & 10.9 & 15 & 13.6 & 14 & 12.7 & 41 & 37.3 \\
Total & 33 & 30 & 14 & 12.7 & 12 & 10.9 & 37 & 33.6 \\
\hline
\end{tabular}

\begin{tabular}{ccc} 
& $\mathrm{N}=110$ & \\
\hline Valor & & Sig. \\
0.236 & & $0.041^{*}$ \\
\hline
\end{tabular}

Nota: Población de 110 mujeres.

$*<0.05$, Correlación significativa

$* *<0.01$, Correlación altamente significativa

El cuadro 3 muestra un valor de 0.236 y una significancia bilateral de 0.041 lo que evidencia una correlación significativa entre ambas variables. El apego parental está relacionado con la dependencia emocional. 


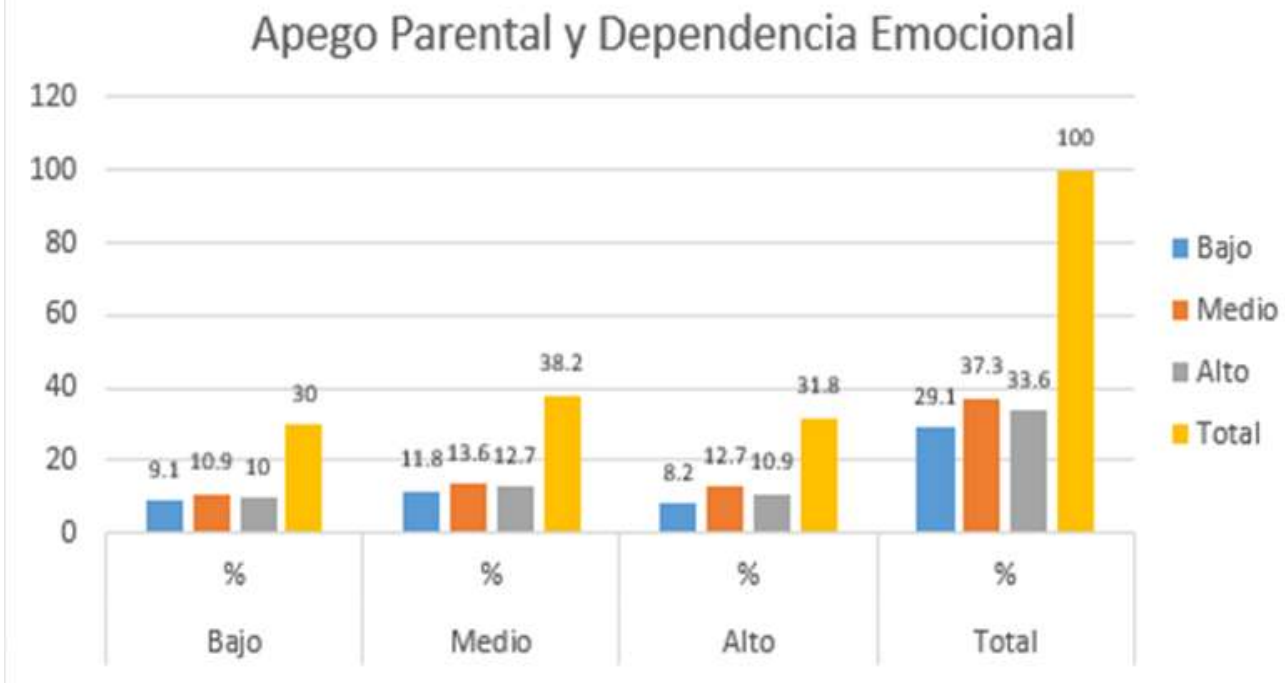

Gráfico 4. Apego parental y dependencia emocional

\section{Correlaciones}

A continuación se presentan los resultados que permiten comprobar o no la hipótesis de investigación:

Hi1. Existe relación entre la dimensión cuidado materno de apego parental y cada una de las dimensiones de dependencia emocional: miedo a la ruptura, miedo e intolerancia a la soledad, prioridad de la pareja, necesidad de acceso a la pareja, deseos de exclusividad, subordinación y sumisión, deseo de control y dominio en mujeres del centro poblado "La Raya" del distrito de Túcume - Lambayeque.

Según los resultados de la correlación de Pearson existe relación entre:

A. El cuidado materno de apego parental y la dimensión Miedo a la ruptura de dependencia emocional.

Hi2. Existe la relación entre la dimensión cuidado paterno de apego parental y cada una de las dimensiones de dependencia emocional: miedo a la ruptura, miedo e intolerancia a la soledad, prioridad de la pareja, necesidad de acceso a la pareja, deseos de exclusividad, subordinación y sumisión, deseo de control y dominio en mujeres del centro poblado "La Raya" del distrito de Túcume - Lambayeque.

B. El cuidado paterno de apego parental y la dimensión prioridad de la pareja de dependencia emocional

Hi3. Existe relación entre la dimensión sobreprotección materna de apego parental y cada una de las dimensiones de dependencia emocional: miedo a la ruptura, miedo e intolerancia a la soledad, prioridad de la pareja, necesidad de acceso a la pareja, deseos de exclusividad, subordinación y sumisión, deseo de control y dominio en mujeres del centro poblado "La Raya" del distrito de Túcume - Lambayeque.

C. Control materno de apego parental y la dimensión miedo a la soledad de dependencia emocional

Hi4. Existe relación entre la dimensión sobreprotección paterna de apego 
parental y cada una de las dimensiones de dependencia emocional: miedo a la ruptura, miedo e intolerancia a la soledad, prioridad de la pareja, necesidad de acceso a la pareja, deseos de exclusividad, subordinación y sumisión, deseo de control y dominio en mujeres del centro poblado "La Raya" del distrito de Túcume - Lambayeque.
D. Control paterno de apego parental y la dimensión prioridad de la pareja de dependencia emocional

E. Control paterno de apego parental y la dimensión subordinación y sumisión de dependencia emocional

F. Control paterno de apego parental y la dimensión deseo de control y dominio de dependencia emocional.

\section{Relación entre cuidado materno de apego parental y miedo a la ruptura de dependencia emocional.}

Cuadro 4. Análisis de relación entre el indicador cuidado materno de apego parental y la dimensión miedo a la ruptura de dependencia emocional.

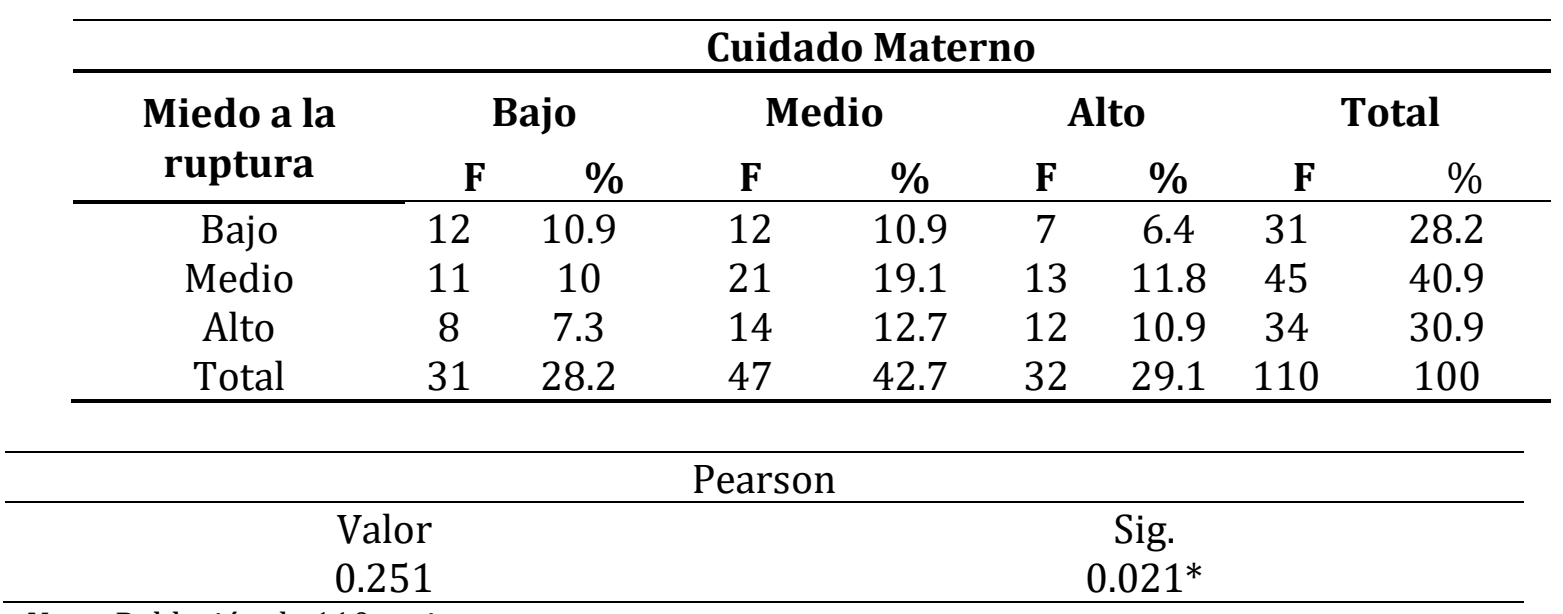

Nota: Población de 110 mujeres.

$*<0.05$ : Correlación significativa

$* *<0.01$ : Correlación altamente significativa

En el cuadro 4 se encontró un valor de 0.251 y una significancia bilateral de 0.021 lo que evidencia una correlación significativa entre ambas variables.

5. Cuidado materno de apego parental y la dimensión miedo a la soledad de dependencia emocional el análisis resultó que no hay correlación estadísticamente significativa. Se encontró un valor de 0.115 y una significancia bilateral de 0.217

6. El Análisis de relación entre el indicador cuidado materno de apego parental y la dimensión prioridad de la pareja de dependencia emocional se encontró un valor de 0.094 y una significancia bilateral de 0.535 , no existe correlación estadísticamente significativa.

7. Análisis de relación entre el indicador cuidado materno de apego parental y la dimensión necesidad de acceso a la pareja de dependencia emocional. Se encontró un valor de 0.221 y una significancia bilateral de 0.088 , no existe correlación estadísticamente significativa. 
8. Análisis de relación entre el indicador cuidado materno de apego parental y la dimensión deseos de exclusividad de dependencia emocional. Se encontró un valor de -0.005 y una significancia bilateral de 0.972 , no existe correlación significativa.

9. Análisis de relación entre el indicador cuidado materno de apego parental y la dimensión subordinación y sumisión de dependencia emocional. Se encontró un valor de 0.057 y una significancia bilateral de 0.657 , no existe correlación estadísticamente significativa.

10. Análisis de relación entre el indicador cuidado materno de apego parental y la dimensión deseo de control y dominio de dependencia emocional. Se encontró un valor de 0.103 y una significancia bilateral de 0.433 , no existe correlación estadísticamente significativa.

11. Análisis de relación entre el indicador cuidado paterno de apego parental y la dimensión miedo a la ruptura de dependencia emocional. Se encontró un valor de 0.081 y una significancia bilateral de 0.554 , no existe correlación estadísticamente significativa.

12. Análisis de relación entre el indicador cuidado paterno de apego parental y la dimensión miedo a la soledad de dependencia emocional. Se encontró un valor de -0.042 y una significancia bilateral de 0.758 , no existe correlación estadísticamente significativa.

13. Análisis de relación entre el indicador cuidado paterno de apego parental y la dimensión prioridad de la pareja de dependencia emocional. Se encontró un valor de 0.296 y una significancia bilateral de 0.002 esto evidencia que existe correlación altamente significativa entre ambas variables.

14. Análisis de relación entre el indicador cuidado paterno de apego parental y la dimensión necesidad de acceso a la pareja de dependencia emocional. Se encontró un valor de -0.146 y una significancia bilateral de 0.271 , no existe correlación estadísticamente significativa.

15. Análisis de relación entre el indicador cuidado paterno de apego parental y la dimensión deseos de exclusividad de dependencia emocional. Se encontró un valor de 0.271 y una significancia bilateral de 0.177 , no existe correlación estadísticamente significativa.

16. Análisis de relación entre el indicador cuidado paterno de apego parental y la dimensión subordinación y sumisión de dependencia emocional. Se encontró un valor de -0.087 y una significancia bilateral de 0.587 , no existe correlación estadísticamente significativa.

17. Análisis de relación entre el indicador cuidado paterno de apego parental y la dimensión deseo de control y dominio de dependencia emocional. Se encontró un valor de -0.036 y una significancia bilateral de 0.774 , no existe correlación estadísticamente significativa.

\section{Control - Apego parental}

18. Análisis de relación entre el indicador control materno de apego parental y la dimensión miedo a la ruptura de dependencia emocional. Se encontró un valor de 0.258 y una significancia bilateral de 0.081 , la correlación no es estadísticamente significativa.

20. Análisis de relación entre el indicador control materno de apego parental y la dimensión miedo a la soledad de dependencia emocional. Se encontró un valor de 0.333 y una significancia 
bilateral de 0.004 lo que evidencia que existe una correlación altamente significativa entre las variables.

21. Análisis de relación entre el indicador control materno de apego parental y la dimensión prioridad de la pareja de dependencia emocional. Se encontró un valor de 0.159 y una significancia bilateral de 0.247 , la correlación no es estadísticamente significativa.

22. Análisis de relación entre el indicador control materno de apego parental y la dimensión necesidad de acceso a la pareja de dependencia emocional. Se encontró un valor de 0.019 y una significancia bilateral de 0.882, la correlación no es estadísticamente significativa.

23. Análisis de relación entre el indicador control materno de apego parental y la dimensión deseos de exclusividad de dependencia emocional. Se encontró un valor de 0.003 y una significancia bilateral de 0.931, la correlación no es estadísticamente significativa.

24. Análisis de relación entre el indicador control materno de apego parental y la dimensión subordinación y sumisión de dependencia emocional. Se encontró un valor de 0.041 y una significancia bilateral de 0.694 , la correlación no es estadísticamente significativa.

25. Análisis de relación entre el indicador control materno de apego parental y la dimensión deseo de control y dominio de dependencia emocional. Se encontró un valor de 0.135 y una significancia bilateral de 0.298 , la correlación no es estadísticamente significativa.

26. Análisis de relación entre el indicador control paterno de apego parental y la dimensión miedo a la ruptura de dependencia emocional. Se encontró un valor de 0.172 y una significancia bilateral de 0.195 , la correlación no es estadísticamente significativa.

27. Análisis de relación entre el indicador control paterno de apego parental y la dimensión miedo a la soledad de dependencia emocional. Se encontró un valor de 0.084 y una significancia bilateral de 0.517 , la correlación no es estadísticamente significativa.

28. Análisis de relación entre el indicador control paterno de apego parental y la dimensión prioridad de la pareja de dependencia emocional. Se encontró un valor de 0.388 y una significancia bilateral de 0.002 esto evidencia que existe correlación altamente significativa entre ambas variables

29. Análisis de relación entre el indicador control paterno de apego parental y la dimensión necesidad de acceso a la pareja de dependencia emocional. Se encontró un valor de 0.094 y una significancia bilateral de 0.268, la correlación no es estadísticamente significativa.

30. Análisis de relación entre el indicador control paterno de apego parental y la dimensión deseos de exclusividad de dependencia emocional. Se encontró un valor de 0.041 y una significancia bilateral de 0.809 la correlación no es estadísticamente significativa.

31. Análisis de relación entre el indicador control paterno de apego parental y la dimensión subordinación y sumisión de dependencia emocional. Se encontró un valor de 0.027 y una significancia bilateral de 0.742 lo que evidencia existe una correlación altamente significativa entre las dimensiones.

32. Análisis de relación entre el indicador control paterno de apego parental y la dimensión deseo de control y dominio de dependencia emocional. Se encontró 
un valor de 0.385 y una significancia bilateral de 0.007 lo que evidencia existe una correlación altamente significativa entre las dimensiones.

En resumen se encontró correlación altamente significativa entre la dimensión afecto paterno la dimensión prioridad de la pareja; control materno con miedo a la soledad; control paterno con las dimensiones prioridad a la pareja, deseos de control y dominio, subordinación y sumisión a un nivel de significancia $(\mathrm{p}<0.01)$. Esto indica que las mujeres del centro poblado "La Raya" presentaron un inadecuado cuidado y afecto en sus figuras de vínculo primario disminuyendo así su autonomía posterior en sus diversas relaciones interpersonales. Tal como menciona (Melis, 2011), la sobreprotección genera en la persona un tipo de apego ansioso resistente, tal condición se manifestará creando un grado de incertidumbre importante en la relación interpersonal futura.

Los resultados de esta investigación coinciden con los resultados obtenidos por Ojeda (2012), en su estudio dependencia emocional y la aceptación de la violencia doméstica, obteniendo puntuaciones altas en ansiedad de separación con un 46,5\%, expresión afectiva de la pareja con un 55,4\% y miedo a la soledad que corresponde al $59,9 \%$. De lo cual se concluye que la población femenina que acuden a la comisaría, presentan niveles altos de dependencia emocional. Los tipos de violencia que han recibido son de tipo psicológica, física $\mathrm{y}$ sexual recalcando que existe una gran influencia de la dependencia emocional en la aceptación de la violencia doméstica. Castelló (2005) explica que la vida para el dependiente emocional será un calvario que puede llegar a límites extremos según el carácter de su pareja, ya que, en muchas ocasiones se propician la aparición de violencia psíquica y física. Si la persona tiene una dependencia emocional grave, aceptará agresiones, humillaciones, infidelidades continuas, burlas, menosprecios.

Así mismo Espíritu (2013). En su investigación dependencia emocional en mujeres violentadas y no violentadas, indica que existen diferencias altamente significativas $82,2 \%$ en el nivel alto y muy alto ( $\mathrm{p}=0,00<0,01$ ); en contraposición de un $5,1 \%$ en el nivel bajo y muy bajo de la dependencia emocional en mujeres no violentadas. Sin embargo en cuanto a los niveles y dimensiones de la dependencia emocional en mujeres violentadas encontró que los puntajes más altos se encuentran en las dimensiones miedo a la ruptura $86,3 \%$; prioridad de la pareja 84,9\%; y subordinación y sumisión $80,9 \%$ en un nivel alto y muy alto respectivamente. El dependiente según Aiquipa (2012) presenta temor ante la sola idea de disolución de la relación de pareja. Por ello, se adopta conductas y comportamientos orientados a mantener la relación, sin importar cuan perjudiciales sean para ellas.

Por otro lado difiere con los resultados generales estudiados por More y Senador (2013), en su investigación "El estilo de apego parental y satisfacción con la pareja”, quienes hallaron al estilo de apego más frecuente en el de control sin afecto (31.07\%). Determinando en la investigación que las parejas se muestran satisfechas en su relación. esto podría discernir por la población que al estar próximos a casarse se encontrarían dentro de la etapa del enamoramiento produciéndose una sobrevaloración general de la pareja distorsionando en algunos casos sus méritos y capacidades debido a la admiración hacia su pareja (Castelló 2012). 


\section{CONCLUSIONES}

Las dos variables apego parental y dependencia emocional se ubican en el nivel medio, con un $42 \%$ y un $41 \%$ de la población en estudio respectivamente.

Existe correlación significativa entre las variables apego parental y dependencia emocional.

Se encontró correlación altamente significativa entre la dimensión afecto paterno y la dimensión prioridad de la pareja; dimensión control materno con las dimensión miedo a la soledad; control paterno con las dimensiones prioridad a la pareja, deseos de control y dominio, subordinación y sumisión. Esto indica que las mujeres del centro poblado "La Raya" presentaron un inadecuado cuidado y afecto en sus figuras de vínculo primario disminuyendo así su autonomía posterior en sus diversas relaciones interpersonales.

Se encontró relación significativa en la encontró la dimensión afecto materno y la dimensión miedo a la ruptura.

\section{REFERENCIAS}

Ainsworth, M. S. (1989). Attachments beyond infancy. American Psychologist, 44(4), 709-716

Aiquipa Tello, J. (2012). Diseño y validación del Inventario de Dependencia Emocional - IDE. Revista de Investigación en Psicología.

DOI: 10.15381/rinvp.v15i1.3673

Bowlby (1969). El vínculo afectivo. Buenos Aires: Paidós

Bowlby (1989) Una base segura: Aplicaciones clínicas de una teoría del apego. Buenos Aires: Paidós

Castelló Blasco, J. (2012). Cómo superar la dependencia emocional. España: Editorial Corona Borealis

Castelló, J. (2005). Dependencia emocional. Características y tratamiento. Madrid,
España: Alianza Editorial

Castelló, J. (2010). Análisis del concepto de Dependencia emocional. I Congreso virtual de Psiquiatría. Ponencia

Espíritu, L. (2013) Dependencia emocional en mujeres violentadas y no violentadas de Nuevo Chimbote. Chimbote: Universidad César Vallejo, Lima

Fonagy, P. (2004). Teoría de apego y psicoanálisis. Barcelona: Espax S.A.

Hazan, C., y Shaver, P. (1994). Attachment as an organizational framework for research on close relationships. Psychological Inquiry, 5(1), 1-22

Lafuente, M. (2003). Patrones de apego, pautas de interacción familiar $\mathrm{y}$ funcionamiento cognitivo. De la década de los 70 y los 80.Revista de Psicología General y Aplicada, 53, 165-190

Melis, F. (2011). Estandarización de PBI para Chile. Santiago: Centro Neuropsiquiátrico de Santiago

More, D. y Senador, A. (2013) Estilos de Apego Parental y Satisfacción de pareja en personas que asisten a charlas matrimoniales en un hospital de Ferreñafe. Chiclayo: Universidad Santo Toribio de Mogrovejo

Ojeda G. (2012) La dependencia emocional y la aceptación de la violencia doméstica en la población femenina que acude a la comisaría de la mujer y la familia de la ciudad de Loja. Ecuador: UNL

Parker, G., Tupling, H. y Brown, L. (1979). Parental Bonding Instrument (PBI). Disponible:

http://www.blackdoginstitute.org.au/res earch/tools/index.cfm\#pbi. [Consulta: 2016, Febrero 23]

Zapata, M. 2015. Entrevista 